\title{
Mesures de prévention non pharmacologiques du delirium de réanimation
}

\author{
Non-Pharmacological Prevention Measures for Delirium in Intensive Care Unit
}

\author{
F. Crozes $\cdot$ M. Planton $\cdot$ S. Silva $\cdot$ C. Haubertin \\ Reçu le 11 août 2017; accepté le 7 juin 2018 \\ (C) SRLF et Lavoisier SAS 2018
}

Résumé Le delirium est défini par un changement brutal ou rapidement progressif de l'état mental ou une modification de l'humeur associés à une baisse des capacités de concentration, à une désorganisation de la pensée, à une confusion et à une altération du niveau de conscience. L'incidence du delirium en réanimation est variable d'environ 4 à $83 \%$, selon les études. Cela est probablement lié à la variété des outils de mesure employés, au niveau d'entraînement des professionnels de santé établissant ces scores, à la profondeur de la sédation et aux différences de populations étudiées. Son étiologie semble être multifactorielle. Il a été montré que la survenue du delirium a un fort impact sur le pronostic vital et fonctionnel des patients en réanimation, car son incidence est associée à une augmentation de la mortalité hospitalière précoce et tardive, et le déclin cognitif qui lui est associé peut persister à distance du séjour en réanimation. Il est important de souligner que la prise en charge dans les soins critiques est très hétérogène. Néanmoins, de nouvelles données de la littérature apportent des éléments concrets sur la prise charge de ce syndrome et fournissent un guide utile à la pratique paramédicale dans la prévention et le dépistage de ce trouble cognitif. L'objectif de ce travail est d'apporter une synthèse autour de la littérature disponible dans ce domaine, mettant en lumière le rôle clé de la profession paramédicale dans ce contexte afin d'identifier des éléments diagnostiques et thérapeutiques susceptibles de modifier pertinemment les pratiques soignantes.

F. Crozes $(\bowtie) \cdot$ S. Silva $\cdot$ C. Haubertin

Critical Care Unit, University Teaching Hospital of Purpan,

F-31059 Toulouse, France

e-mail : crozes.f@chu-toulouse.fr

M. Planton

Department of Neurology, Toulouse University Hospital,

F-31059 Toulouse, France

M. Planton · S. Silva

Toulouse NeuroImaging Center, Toulouse University, Inserm 1214, UPS, F-31000 Toulouse, France
Mots clés Delirium · Réanimation · Traitements non pharmacologiques

Abstract Delirium is defined as an abrupt or rapid change in mental status or mood, having difficulties with concentration and general disorder in ability to think, as well as experiencing confusion and altered consciousness. The incidence of delirium in intensive care units varies from about 4 to $83 \%$. This difference can be attributed to reasons linked with various levels of training of the health professionals establishing the scores, the depth of sedation, and the differences in samples studied. Its etiology is clearly multifactorial. It has been shown that the occurrence of delirium has a strong impact on the vital and functional prognosis of patients in resuscitation, associated with an increase in early and late hospital mortality as well as a cognitive decline which may persist beyond the stay in the intensive care unit. It is important to note that the critical care serviced to patients experiencing delirium is extremely heterogeneous. However, new data from literature provides concrete ways to manage this syndrome and proposes a guide to paramedical practice for the prevention and screening of this cognitive disorder. The objective of this work is, therefore, to synthesize the literature available in this field, highlighting the key role of the paramedical profession in this context in identifying the diagnostic and therapeutic elements to put in practice, and so to modify care practices.

Keywords Delirium · ICU · Non-Pharmacological interventions

\section{Introduction}

Ce travail a pour but de présenter une revue de la littérature ayant pour thème le delirium des patients de soins intensifs, en détaillant de façon la plus exhaustive possible les mesures de prévention non pharmacologiques actuelles. Il définit 
dans un premier lieu la problématique, différents outils diagnostiques, l'épidémiologie (incidence, facteurs de risque et conséquences) et enfin des techniques de prévention avec les difficultés de mise en œuvre.

\section{Définition}

Le delirium est décrit selon l'Association américaine de psychiatrie dans le Manuel de classification diagnostique depuis la version IV du DSM (Diagnostic and Statistical Manual) [1]. Il est défini par une perturbation de la conscience, accompagnée de modifications cognitives qui ne peuvent pas s'expliquer par une démence préexistante ou en évolution. La perturbation s'installe en un temps court, habituellement quelques heures ou quelques jours, et tend à avoir une évolution fluctuante tout au long de la journée.

Les troubles liés au delirium ont des caractéristiques communes, mais les étiologies peuvent être différentes. En effet, nous retrouvons quatre étiologies au delirium dans la littérature actuelle : le delirium, dû à une affection médicale générale, induit par une substance (incluant les effets secondaires des médicaments), dû à des étiologies multiples et enfin le delirium non spécifié, c'est-à-dire lorsque le clinicien est dans l'impossibilité de déterminer une étiologie spécifique au delirium. La notion de diagnostic différentiel, par élimination d'une étiologie, est essentielle dans l'identification de ce syndrome.

Plusieurs termes ont été utilisés pour décrire ce syndrome, tels que psychose des unités de soins intensifs, état confusionnel aigu, encéphalopathie, dysfonctionnement cérébral aigu [2]. Néanmoins, la littérature récente a uniformisé cette sémiologie et utilise actuellement le concept syndromique de delirium.

\section{Signes cliniques}

Depuis le DSM-IV, les caractéristiques diagnostiques du delirium sont décrites selon trois critères que l'on retrouve comme suit dans la version française du manuel (p. 158-159) :

« Critère A : la perturbation de la conscience se manifeste par une baisse de l'état de conscience de l'environnement. La capacité à focaliser, soutenir ou mobiliser l'attention est altérée. Il faut répéter les questions au sujet parce qu'il ne peut pas fixer son attention ; parfois, il persévère à répéter la réponse à une question précédente au lieu de déplacer son attention. Le sujet est facilement distrait par des stimuli hors de propos. Il peut être difficile (voire impossible) d'engager une conversation avec lui en raison de ces perturbations.

Critère B : on observe également des modifications du fonctionnement cognitif (qui peuvent inclure une altération de la mémoire, une désorientation, une perturbation du langage) ou bien l'apparition de perturbations des perceptions. L'altération de la mémoire se manifeste le plus généralement par des troubles de la mémoire récents qui peuvent être évalués en demandant au sujet de se souvenir des noms de différents objets sans relation entre eux ou d'une phrase courte puis ensuite de les répéter après avoir distrait son attention pendant quelques minutes. La désorientation se manifeste habituellement dans le temps ou dans l'espace. Dans les formes légères du delirium, la désorientation dans le temps peut être le premier symptôme à apparaître. La nonreconnaissance de soi-même est plus rare. La perturbation du langage peut apparaitre clairement sous forme d'une dysarthrie, d'une dysnomie ou d'une dysgraphie, ou même d'une aphasie. Parfois, le discours est décousu et hors de propos ; d'autre fois, il est précipité et incohérent avec des changements de sujet imprévisibles.

Le clinicien peut éprouver des difficultés à évaluer les modifications cognitives en raison de l'inattention et de l'incohérence du sujet. Dans ces circonstances, il est utile de reprendre en détail l'histoire de la maladie et de rechercher d'autres sources d'informations que le sujet lui-même, en particulier auprès des membres de sa famille.

Les anomalies des perceptions peuvent comprendre des interprétations erronées, des illusions ou des hallucinations. Par exemple, le claquement d'une porte peut être pris pour un coup de feu (interprétation erronée), les plis des draps pour des objets animés (illusion) ou bien l'individu peut "voir" un groupe de personnes planant au-dessus de son lit alors qu'il n'y a en réalité personne (hallucination). Bien que les perceptions sensorielles erronées soient le plus souvent visuelles, elles peuvent aussi concerner d'autres modalités sensorielles comme l'audition, le toucher, le goût et l'olfaction. Les perceptions erronées vont des plus simples et uniformes aux plus complexes. L'individu peut être convaincu de la réalité de ses hallucinations et avoir des réactions émotionnelles et comportementales en rapport avec leur contenu.

Critère $C$ : la perturbation s'installe en un temps court et tend à avoir une évolution fluctuante tout au long de la journée. Par exemple, la personne peut être cohérente et coopérante pendant la visite à l'hôpital le matin, et le soir même exiger qu'on retire sa perfusion, afin qu'elle rentre chez elle pour voir des parents décédés depuis des années. »

Par ailleurs, on peut souligner que dans les caractéristiques et troubles associés, il existe fréquemment une perturbation $\mathrm{du}$ cycle veille-sommeil. Le comportement psychomoteur est fréquemment perturbé. On retrouve trois formes distinctes, un comportement psychomoteur non modifié qui est qualifié de forme normoactive, d'autres individus sont agités et présentent une forme hyperactive. Cette hyperactivité motrice peut comporter des gestes de tâtonnement ou de grattage des draps, des tentatives dangereuses ou intempestives pour sortir du lit, des mouvements brusques. À l'opposé, il peut y 
avoir une diminution de l'activité motrice (forme hypoactive), avec une lenteur et une léthargie confinant à la stupeur.

L'activité psychomotrice peut passer d'un extrême à l'autre au cours de la journée. Les troubles du jugement peuvent entraver l'observance du traitement. Hallucinations, idées délirantes et agitation sont plus fréquentes dans les phases d'hyperactivité. Hallucinations, idées délirantes et autres troubles perceptifs sont moins fréquents dans les phases d'hypoactivité. Le degré d'altération du fonctionnement cognitif observé dans les états d'hyperactivité et d'hypoactivité est comparable.

\section{Outils diagnostiques}

Pour dépister le delirium, plusieurs outils ont été développés au cours des dernières années; l'échelle RADAR qui permet de reconnaître un delirium aigu dans la pratique courante [3] ou l'échelle de confusion NEECHAM [4], ou le score DDS [5].

Deux échelles d'évaluation de la confusion ont été validées pour permettre au personnel soignant non formé à la psychiatrie de dépister le delirium. Le Confusion Assessment Method for the Intensive Care Unit (CAM-ICU) [6] (Fig. 1) et l'Inten- sive Care Delirium Screening Checklist (ICDSC) [7] (Fig. 2) sont des échelles utilisées dans les unités de soins intensifs. Ces deux échelles semblent se démarquer actuellement. En effet, dans une étude récente [8], 210 patients ont été évalués simultanément avec la CAM-ICU, l'ICDSC, et les résultats ont été comparés avec l'outil de référence DSM-IV-TR. La prévalence du delirium était variable selon l'échelle utilisée ; cependant, la CAM-ICU a montré une sensibilité de $50 \%$ et une spécificité élevée de $95 \%$. L'ICDSC a également une bonne valeur diagnostique avec une sensibilité de $63 \%$ et une spécificité de $95 \%$. Avec une validité de corrélation identique (Cohen $\kappa$ : $[0,41-0,60])$ pour ces deux échelles, l'ICDSC présente une meilleure sensibilité (Tableau 1).

Par ailleurs, la référence du diagnostic du delirium est le DSM dont les critères sont difficilement évaluables par un infirmier dans sa pratique courante. L'utilisation du DSM comme outil diagnostique n'est pas reproductible dans toutes les réanimations, car il nécessiterait le recours à un psychiatre sur des amplitudes horaires de 24 heures. Une des difficultés majeures du diagnostic réside dans le fait que les différents outils diagnostiques (CAM-ICU-ICDSC) sont sensibles et spécifiques à tous les états confusionnels, qu'ils soient d'origines neurologiques, psychiatriques ou autres : syndrome méningé, hypertension intracrânienne, déficit cortical, délire

Organigramme de la méthode d'évaluation de la confusion mentale pour la réanimation (CAM-ICU)

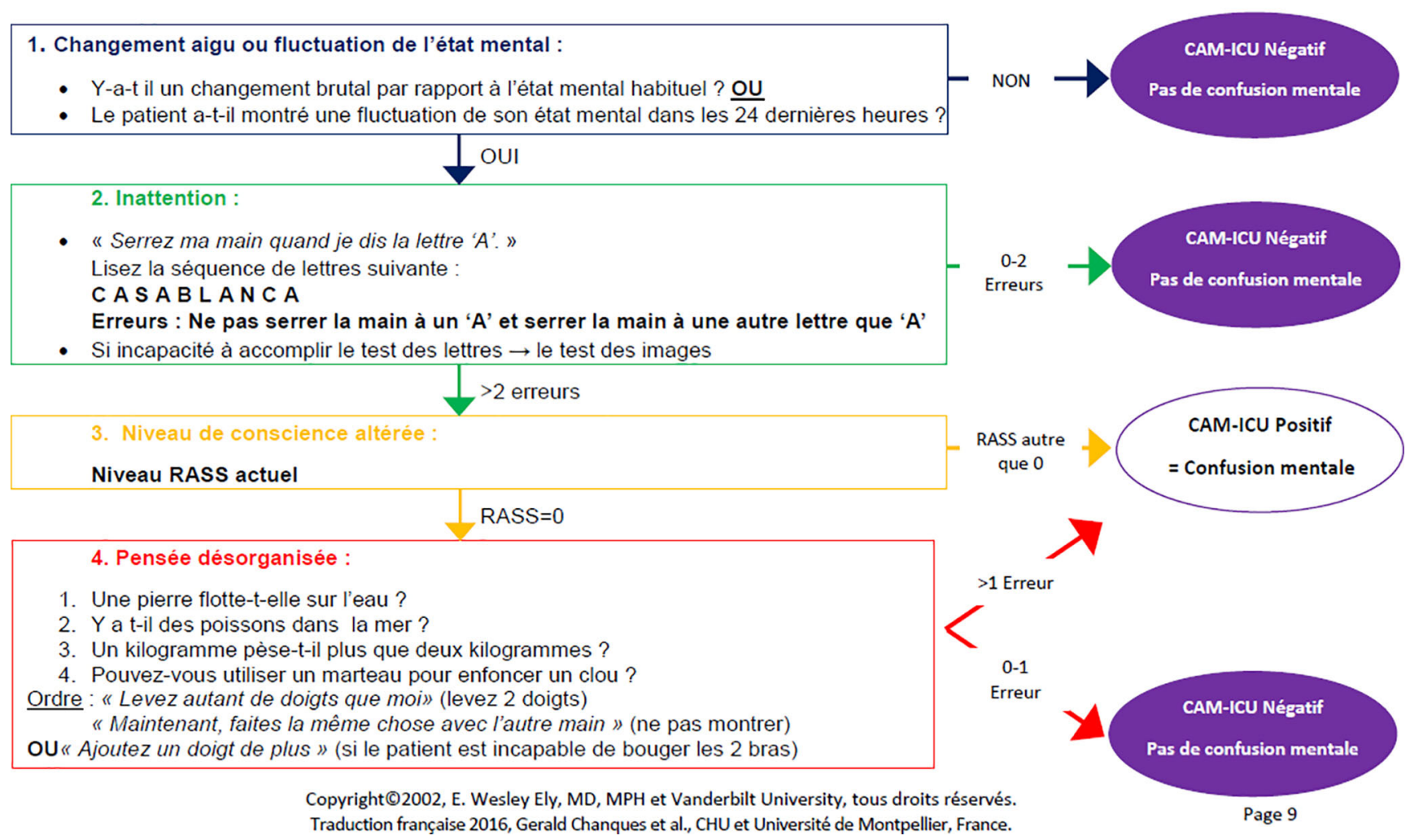

Fig. 1 CAM-ICU 


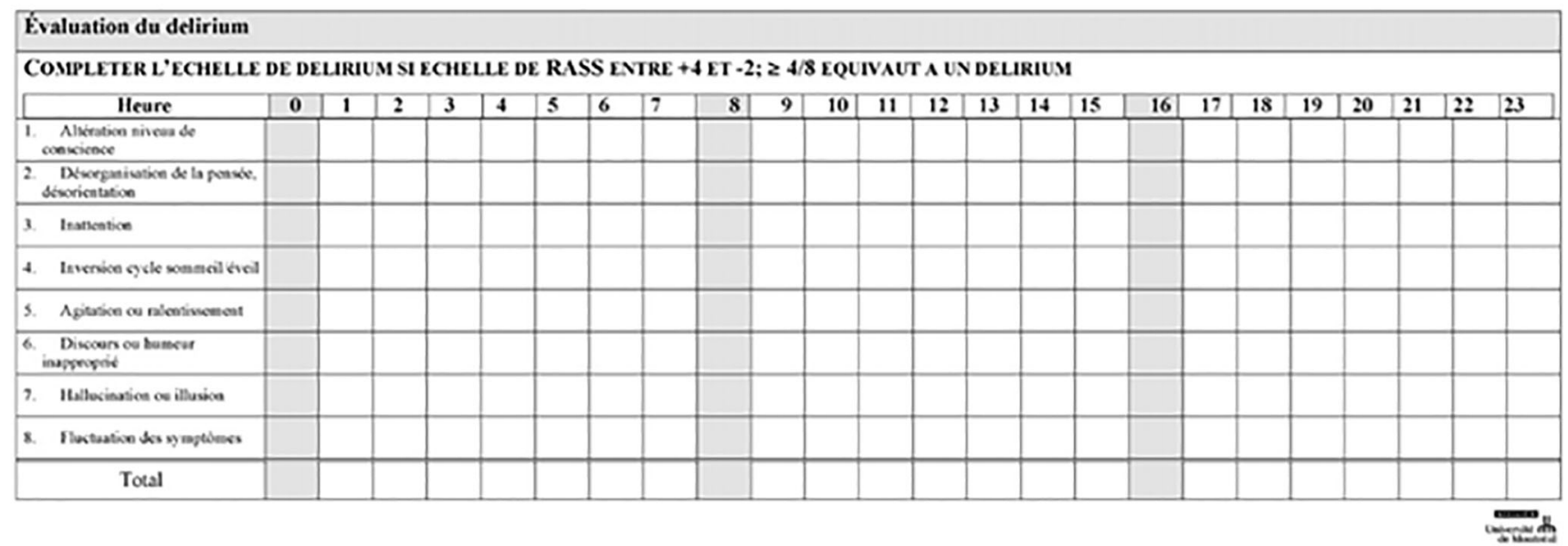

\section{Échelle d'évaluation:}

1. Altération du niveau de conscience :

- réponse exagérée à stimulation normale (ex: sursaute exagérément à un timbre de voix normal)

- somnolence ou nócessité de stimulation modḱréc pour obtenir réponse

2. Désorganisation de la pensée, orientation :

- incapacité de répondre à des questions simples et directes, nécessitant un oui ou un non (ex. : étes-vous à l'église?, sommes-nous en automne?)

- incapacité de répondre correctement aux exemples de questions suivantes (ex. : Une roche flotte-t-elle sur l'eau? Y a-t-il des poissons dans la mer?)

3. Inattention :

- difficulté à exćcuter une suite de 2 ordres simples (ex : Levez la main droite, puis le pied gauche)

- dificulté à suivre une conversation simple en gardant un contact visuel

- facilement distrait par stimuli extérieurs

- difficulté à ramener son attention

4. Inversion du cycle sommeil/éveil :

- dort moins de 4 heures par nuit

- éveils fréquents indépendants de ceux provoqués par le personnel

- agité la nuit, dort le jour

5. Agitation ou ralentissement :

- agitation psychomotrice nécessitant $\uparrow$ des doses de sédatifs et/ou contentions

- ralentissement psychomoteur en état d'éveil

6. Discours ou humeur inappropriće:

- propos incohérents ou inappropriés (ex. : désinhibition sexuelle)

- humeur ou émotion non reliće à la situation actuelle (ex. : éclat de rire ou pleurs spontanés sans raison extérieure, instabilité émotionnelle)

7. Hallucinations ou illusion :

- manifestations cliniques évidentes d'hallucinations (ex. : essaie d'attraper objets qui n'existent pas ou entend des voix qui n'existent pas)

- interprétation erronée de la réalité (ex. : l'alarme de pompe à soluté est interprétée comme une sonnette de porte, propos hostiles ou paranoildes)

8. Fluctuation des symptômes :

alternance des manifestions observables au cours d'une mème journée (ex. : attentif et orienté à un moment, puis désorienté à un autre)

Fig. 2 ICDSC

paranoïaque, psychose puerpérale, AVC, méningite, intoxication, traumatisme et bien d'autres. En effet, ils se caractérisent tous par une désorganisation des processus de la pensée qui est l'un des symptômes communs avec le delirium.
Aucun signe pathognomonique n'est retrouvé dans le delirium, son identification se fait donc par un diagnostic d'exclusion des différentes affections entraînant une altération cognitive. 
Tableau 1 Validité de corrélation, sensibilité et spécificité, valeur prédictive positive et négative (VPP et VPN) de CAM-ICU et ICDSC par rapport aux critères de diagnostic DSM-IV-TR [8]

\begin{tabular}{|c|c|c|c|c|c|c|c|c|c|c|}
\hline & $\kappa$ & $\rho$ & Sensitivity & $\mathrm{CI}_{95 \%}$ & Specificity & $\mathrm{CI}_{95 \%}$ & PPV & $\mathrm{CI}_{95 \%}$ & NPV & $\mathrm{CI}_{95 \%}$ \\
\hline $\begin{array}{l}\text { DSM-IV-TR } \\
\text { and CAM-ICU }\end{array}$ & 0,44 & $<0,001$ & 46,7 & $39,3-57,4$ & 94,9 & $89,3-98,1$ & 87,8 & $75,2-95,4$ & 69,6 & $61,8-76,6$ \\
\hline $\begin{array}{l}\text { DSM-IV-TR } \\
\text { and ICDSC }\end{array}$ & 0,60 & $<0,001$ & 63 & $52,3-78,9$ & 94,9 & $89,3-98,1$ & 90,6 & $80,7-96,5$ & 76,7 & $69-88,8$ \\
\hline $\begin{array}{l}\text { CAM-ICU and } \\
\text { ICDSC }\end{array}$ & 0,56 & $<0,001$ & 77,6 & $63,4-88,2$ & 84,4 & $77,2-59,2$ & 59,4 & $46,4-71,5$ & 92,5 & $86,9-96,2$ \\
\hline
\end{tabular}

\section{Épidémiologie}

\section{Incidence}

L'incidence du delirium de réanimation, rapportée dans les études de cohorte dans les services de réanimation médicaux et chirurgicaux, varie entre 3,6\% [2] et 83,3\% [9]. Cette variabilité s'explique par la divergence des outils de mesure, le niveau d'entraînement des professionnels de santé établissant ces scores et les différences de populations étudiées [10].

\section{Facteurs de risque}

Son étiologie semble être multifactorielle [11], associant des éléments liés à la pathologie (par exemple sepsis), le terrain du patient, les traitements utilisés, mais aussi des facteurs environnementaux souvent oubliés dans ce contexte [12,13].

En effet, la physiopathologie du delirium n'est pas précisément établie, mais plusieurs pistes font l'objet de recherches, impliquant des interactions complexes entre inflammation, neurotransmission et système nerveux autonome au niveau cérébral [14].

Parmi les principaux facteurs de risque identifiés dans la littérature, nous retrouvons :

- la durée de sédation et la durée de ventilation [15] ;

- l'âge [2] ;

- l'altération du cycle nycthéméral [16] ;

- la gravité de la pathologie ayant justifié l'admission en réanimation [17].

Le dépistage et la connaissance sur l'étiologie du delirium sont étroitement liés et vont conditionner la qualité de la prise en charge de cette pathologie. Van den Boogaard a développé en 2012 un modèle prédictif du delirium chez les patients de réanimation. Son modèle se fonde sur dix facteurs de risque qui sont l'âge, le score Apache II, le type d'admission, le coma, l'infection, les lactates, l'utilisation d'anesthésiants et de morphiniques, le taux d'urée et l'admission en urgence. Force est de constater que la plupart des facteurs de risque ne sont pas contrôlables comme l'âge ou le type d'admission ; mais d'autres, comme la sédation administrée, sont modifiables et peuvent potentiellement améliorer le caractère prédictif de la maladie [18].

De multiples facteurs d'inconfort sont reconnus facteurs déclenchants, notamment la douleur [19] et le manque de sommeil [20,21]. La soif [22], l'anxiété [23], le bruit [12, $13]$ ont également été décrits comme facteurs favorisants.

\section{Impact vital et fonctionnel}

La survenue d'un épisode de delirium au cours d'une hospitalisation est associée à un pronostic fonctionnel et cognitif plus sévère. Il provoque une augmentation de la mortalité en réanimation ainsi qu'à distance de l'épisode [24]. Il engendre une augmentation de la durée d'hospitalisation et donc un surcoût hospitalier significatif [25].

De manière intéressante, il a été décrit une altération cognitive chez $24 \%$ des patients survivant à un syndrome de détresse respiratoire aiguë, persistant jusqu'à six ans après l'épisode [26]. Cette altération est associée à une difficulté de réhabilitation à domicile et/ou à l'apparition d'un syndrome de stress post-traumatique. Ces éléments ont un impact direct sur la qualité de vie et génèrent une souffrance psychique lors de son retour à domicile.

L'impact du delirium sur le fonctionnement cognitif et son évolution n'ont été que très peu étudiés. La plupart des études ont mesuré la cognition avec des tests d'évaluation globale comme le Mini-Mental State Examination [27,28]. Ces dernières études ont montré une diminution de l'efficience intellectuelle globale chez les patients dans les jours qui suivaient l'hospitalisation, mais sans caractériser un profil neuropsychologique. Une étude s'est attachée à évaluer de façon exhaustive le fonctionnement cognitif de 
821 patients à 3 et 12 mois d'une hospitalisation en soins intensifs de chirurgie médicale. Bien que $6 \%$ des patients présentaient des troubles cognitifs en phase aiguë, à 12 mois, un tiers des patients montraient une altération cognitive. La durée du delirium a été indépendamment associée à une diminution du fonctionnement cognitif global et exécutif à 12 mois [29]. Nous retrouvons également une revue de la littérature récente [30] qui identifie une prévalence plus élevée de syndrome dépressif pour les patients ayant eu un delirium, le lien avec le syndrome de stress post-traumatique n'a pas pu être conclu au vu du peu d'études menées sur le sujet.

Aucun profil type n'a pu être réellement détaillé. Les conséquences sur le plan psychocomportemental ou encore les répercussions des troubles cognitifs sur la qualité de vie mériteraient d'être plus explorées à travers une étude de méthodologie plus robuste au vu des éléments que suggère la littérature sur une potentielle causalité que pourrait être le delirium.

\section{Prévention}

On a pu constater que le delirium a des étiologies multifactorielles. Il n'est pas possible d'intervenir sur tous les facteurs de risque tels que l'âge, la gravité de la pathologie initiale, le type de chirurgie. Toutefois, il est possible d'intervenir sur certains facteurs comme la douleur ou la durée de la sédation, permettant d'améliorer le pronostic de la maladie.

On retrouve dans la littérature plusieurs moyens de prévention du delirium. Au niveau pharmacologique, une molécule, la dexmédétomidine, a montré son efficacité sur l'incidence du delirium chez des patients au cours de leur hospitalisation en soins intensifs de deux hôpitaux de Beijing [31]. Des conclusions similaires ont également été retrouvées chez des patients de soins intensifs sous ventilation mécanique, présentant un delirium, hospitalisés sur raisons médicales et chirurgicales à Melbourne [32].

\section{Recommandations et données de la littérature}

De nombreuses actions de prévention non pharmacologique sont également décrites. Certaines sociétés savantes faisant référence en soins critiques comme The American College of Critical Care Medicine émettent des recommandations sur la prise en charge de la douleur, l'agitation et le delirium [33], retrouvées sous l'acronyme «PAD» (Pain, Agitation and Delirium) sur les bases de données anglaises. L'application de ces recommandations constitue un exemple de l'implication des infirmières dans l'élaboration et la mise en œuvre de lignes directrices professionnelles dans la prise en charge de la douleur, de l'agitation et du delirium [34]. Le PAD propose des actions claires au niveau préventif, curatif ainsi que des échelles de dépistage et d'évaluation sur chacun des trois versants (douleur, agitation et delirium) (Fig. 3).
La mise en application d'un groupe d'actions coordonnées appelé ABCDEF bundle [35] a montré une meilleure survie, une diminution du nombre de jours de delirium et de coma. La mise en pratique de cet acronyme consiste en l'application d'actions coordonnées :

- évaluation et prise en charge de la douleur (asses, prevent and manage pain);

- coordination du sevrage respiratoire et du réveil (both spontaneous awakening trials and spontaneous breathing trail);

- gestion de la sédation et de l'analgésie (choice of analgesia and sedation);

- évaluation, prévention et prise en charge du delirium (delirium: assess, prevent and manage);

- mobilisation précoce (early mobility and exercise) ;

- implication et participation des familles (family engagement and empowerment).

La méthodologie de l'étude ne permet pas d'isoler une action ayant une supériorité d'efficacité dans la diminution du nombre de jours de delirium. L'action « évaluation, prévention et prise en charge du delirium » consiste en son évaluation, sa prévention et son traitement. La prévention du delirium reprend différentes actions avec l'identification des facteurs de risque, la diminution des benzodiazépines, la réalisation d'exercice par le patient, la promotion du sommeil. On retrouve donc dans la partie « évaluation, prévention et prise en charge du delirium » du bundle des interactions directes avec les autres mesures. Par exemple, il y a une interaction entre l'action « gestion de la sédation et de l'analgésie » du bundle et la prévention du delirium qui propose la diminution des benzodiazépines. Il en est de même pour l'action « mobilisation précoce » et la réalisation d'exercices par le patient dans la prévention du delirium. Les résultats de l'étude soulignent l'importance d'un bundle d'actions associant donc la coordination de l'ensemble des mesures : du réveil et de la ventilation, la gestion de la sédation, le dépistage et la gestion du delirium, la mobilité précoce et l'engagement de la famille.

D'autres études montrent que les bundles multicomposants, avec un plus grand nombre de stratégies visant l'évaluation, la prévention et le traitement du delirium, peuvent améliorer les résultats cliniques [36,37].

\section{Gestion des nuisances environnementales}

Le PAD et l'ABCDEF bundle sont biens décrits au travers des études ; toutefois, la connaissance plus fine des facteurs environnementaux favorisant le delirium permettrait de mettre en place des actions préventives non pharmacologiques. Une étude a montré que l'environnement modifiait la durée du delirium. Zaal et al. montrent, sur leur étude avant/après, que le nombre de jours où les patients présentent un delirium 


\begin{tabular}{|c|c|c|c|}
\hline & PAIN & AGITATION & DELIRIUM \\
\hline II & $\begin{array}{l}\text { Assess pain } \geq 4 x \text { /shift \& prn } \\
\text { Preferred pain assessment tools: } \\
\text { - Patient able to self-report } \rightarrow \text { NRS }(0-10) \\
\text { - Unable to self-report } \rightarrow \text { BPS }(3-12) \\
\text { or CPOT (0-8) } \\
\text { Patient is in significant pain if NRS } \geq 4 \text {, } \\
\text { BPS } \geq 6 \text {, or CPOT } \geq 2\end{array}$ & $\begin{array}{l}\text { Assess agitation, sedation } 24 x / \text { shift } \& \text { prn } \\
\text { Preferred sedation assessment tools: } \\
\text { - RASS }(-5 \text { to }+4) \text { or } S A S(1 \text { to } 7) \\
\text { - NMB } \rightarrow \text { suggest using brain function monitoring } \\
\text { Depth of agitation, sedation defined as: } \\
\text { - agitated if RASS }=+1 \text { to }+4 \text {, or } S A S=5 \text { to } 7 \\
\text { - awake and calm if RASS }=0 \text {, or } S A S=4 \\
\text { - lightly sedated if RASS }=-1 \text { to }-2 \text {, or SAS }=3 \\
\text { - deeply sedated if RASS }=-3 \text { to }-5 \text {, or SAS }=1 \text { to } 2\end{array}$ & $\begin{array}{l}\text { Assess delirium } Q \text { shift \& pm } \\
\text { Preferred delirium assessment tools: } \\
\text { - CAM-ICU (+or -) } \\
\text { - ICDSC }(0 \text { to } 8) \\
\text { Delirium present if: } \\
\text { - CAM-ICU is positive } \\
\text { - ICDSC } \geq 4\end{array}$ \\
\hline & $\begin{array}{l}\text { Treat pain within } 30 \text { " then reassess: } \\
\text { - Non-pharmacologic treatment- } \\
\text { relaxation therapy } \\
\text { - Pharmacologic treatment: } \\
\text { - Non-neuropathic pain } \rightarrow \text { IV opioids } \\
+/ \text { - non-opioid analgesics } \\
\text { - Neuropathic pain } \rightarrow \text { gabapentin or } \\
\text { carbamazepine, + IV opioids } \\
\text {-S/p AAA repair, rib fractures } \rightarrow \\
\text { thoracic epidural }\end{array}$ & $\begin{array}{l}\text { Targeted sedation or DSI (Goal: patient } \\
\text { purposely follows commands without agitation): } \\
\text { RASS }=-2-0, \text { SAS }=3-4 \\
\text { - If under sedated (RASS }>0, \text { SAS }>4 \text { ) } \\
\text { assess/treat pain } \rightarrow \text { treat vi/sedatives prn } \\
\text { (non-benzodiazepines preferred, unless ETOH or } \\
\text { benzodiazepine withdrawal is suspected) } \\
\text { - If over sedated(RASS }<-2, \text { SAS }<3 \text { ) hold } \\
\text { sedatives untl at target, then restart at } \\
50 \% \text { of previous dose }\end{array}$ & $\begin{array}{l}\text { - Treat pain as needed } \\
\text { - Reorient patients; familiarze } \\
\text { surroundings; use patient's eyeglasses, } \\
\text { hearing aids if needed } \\
\text { - Pharmacologic treatment of delirium: } \\
\text { - Avoid benzodiazepines unless ETOH or } \\
\text { benzodiazepine withdrawal is suspected } \\
\text { - Avoid rivastigmine } \\
\text { - Avoid antipsychotics if } \uparrow \text { risk of } \\
\text { Torsades de pointes }\end{array}$ \\
\hline 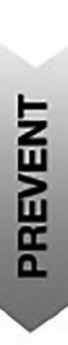 & $\begin{array}{l}\text { - Administer pre-procedural analgesia } \\
\text { and/or non-phamacologic interventions } \\
\text { (e.g., relaxation therapy) } \\
\text { - Treat pain first, then sedate }\end{array}$ & $\begin{array}{l}\text { - Consider daily SBT, early mobility and exercise } \\
\text { when patients are at goal sedation level, unless } \\
\text { contraindicated } \\
\text { - EEG monitoring if: } \\
\text { - at risk for seizures } \\
\text { - burst suppression therapy is indicated for } \uparrow \text { ICP }\end{array}$ & $\begin{array}{l}\text { - Identify delirium risk factors: dementia, } \\
\text { HTN, ETOH abuse, high severity of illness, } \\
\text { coma, benzodiazepine administration } \\
\text { - Avoid benzodiazepine use in those at } \uparrow \\
\text { risk for delirium } \\
\text { - Mobilize and exercise patients early } \\
\text { - Promote sleep (control light, noise; cluster } \\
\text { patient care activities; decrease nocturnal } \\
\text { stimuli) } \\
\text { - Restart baseline psychiatric meds, if } \\
\text { indicated }\end{array}$ \\
\hline
\end{tabular}

Adapted with permlssbn. @ 2013. Wolters Kluwer Health. Barr J, Fraser GL, Puntillo K, et al. Cilnical Practice Guldellnes for the Management of Paln, Agitation and Dellinum In Adult Pattents in the Intenshe Care Unit. Crit Care Med. 2013; 1:263-306.

Fig. 3 Groupe d'actions coordonnées de la prise en charge PAD (Douleur Agitation Delirium) en soins intensifs

est diminué de 0,4 jours sur la phase après, cette phase était mesurée chez des patients hospitalisés en chambres individuelles, suite aux réaménagements des locaux où se déroulait l'étude. La phase avant avait été mesurée chez des patients hospitalisés dans le même service où les lits étaient disposés dans un espace commun, avant la réorganisation des locaux [13]. L'activité continue des soins impose des nuisances telles que le bruit et la lumière. Le défaut de gestion du bruit ainsi que la lumière excessive font augmenter de façon significative le nombre de jours de delirium. Ces nuisances environnementales imposées de façon constante viennent altérer le rythme circadien des patients, le manque de sommeil apparaît également comme facteur favorisant du delirium [20].

Dans son évaluation des sources d'inconfort perçues par les patients, Kalfon et al. questionnent 868 patients. Parmi 16 sources d'inconfort proposées, les scores les plus élevés sont ressentis par les patients pour le manque de sommeil, le fait d'être relié et dépendant de tuyaux (intubation, tubulures et câbles), la douleur et la soif [38]. Une sous-évaluation et une mauvaise gestion de ces sources d'inconfort pourraient favoriser des états d'agitation et de delirium. Cette étude observationnelle met en lumière le vécu de patients et suggère de repenser les soins autour de ces différents axes prioritaires : favoriser sommeil, prendre en soin l'angoisse liée au sentiment de dépendance relative aux tuyaux, améliorer la prise en charge de la douleur et la gestion de la soif de façon systématique.

\section{Communication thérapeutique}

La mise au point de Puntillo et al. vient confirmer ces éléments. La prévalence de symptômes stressants comme la douleur et la soif reste encore sous-évaluée et sous-traitée [39]. La difficulté soulignée reste l'évaluation de ces symptômes chez les patients non communicants. Cette revue de la littérature propose plusieurs approches permettant l'évaluation de cette population et enfin différentes prises en charge non pharmacologiques. Dans la gestion de la soif, l'usage sur les muqueuses buccales de spray d'eau stérile, de gels hydratants, de compresses mouillées ou encore l'emploie d'humidificateurs réchauffeurs pour les patients intubés sont 
proposés. Dans la gestion de la douleur, le massage, la relaxation, la musicothérapie sont proposés. Bien que l'efficacité de ces techniques ne soit pas clairement établie dans la littérature, la gestion de la douleur et des mauvaises expériences vécues du milieu hospitalier par l'hypnose, aujourd'hui fréquemment utilisée sur la phase d'induction au bloc opératoire [40], a bien été objectivée grâce à la neuro-imagerie [41,42]. La modification de l'activité cérébrale induite par l'hypnose a été démontrée sur des volontaires sains ayant eu un PET-scan (tomographie par émission de positons) avant et après induction hypnotique. En effet, on constate sur les imageries qu'il y a une modulation de l'activation du cortex cingulaire antérieur sous hypnose corrélé à une diminution de douleur (évaluée par l'échelle EVA) induite par stimuli chez les patients. L'hypnose permet donc de moduler l'activation cérébrale et ainsi d'agir sur les voies nociceptives.

L'utilisation d'un langage positif doit être envisagée et employée en milieu de soins afin de prendre en charge la douleur et le mauvais vécu des patients de réanimation [43].

\section{Place aux familles}

L'efficacité des soins psychologiques sur la récupération des patients est confirmée par l'étude de Black et al. qui utilisent dans le bras interventionnel un journal de bord ainsi qu'une formation dédiée aux familles sur la façon de parler et de réorienter le patient. Les résultats de l'étude ne permettent pas de conclure que les soins psychologiques diminuent significativement le delirium des patients, mais les patients du bras interventionnel ont une meilleure récupération psychologique et un meilleur bien-être que ceux du bras témoin, et cela, jusqu'à 12 semaines après l'intervention [44].

Avec les progrès de la médecine, de plus en plus de patients « survivent » à la réanimation, ce qui permet d'alimenter des bases de données et de retrouver dans la littérature actuelle les conséquences liées à une hospitalisation en réanimation [45]. Des symptômes sont décrits pour les patients mais concernent également les familles comme le décrivent Huggins et al. En effet, le syndrome de stress post-traumatique retrouvé chez les patients après leur séjour en réanimation peut également toucher les familles, on parlera de syndrome post-soins intensifs de la famille. Une revue de la littérature [46] menée sur des études de 1980 à 2011 combinant le delirium avec « détresse », « souvenir », " anxiété », « dépression », « syndrome de stress posttraumatique » fait un examen qualitatif des expériences vécues lors d'épisodes de delirium. Cette étude met en exergue le vécu des patients se rappelant leur délire dont les souvenirs sont généralement sources d'angoisses, de mal-être et difficilement acceptés. Cette détresse peut également être retrouvée chez les proches témoins et entraîner des séquelles psychologiques à plus long terme.
L'information et la prise en charge des proches peuvent donc réduire la détresse et la morbidité psychologiques. Une première étude de Jabre et al. conduite en 2013 [47] a mesuré l'effet sur la famille de leur présence lors de la réanimation cardiorespiratoire de leur proche. Les résultats montrent, grâce à une interview téléphonique 90 jours après la réanimation, que la détresse émotionnelle ainsi que la présence de symptômes relatifs au syndrome de stress post-traumatique étaient significativement plus sévères dans le groupe témoin que dans le groupe d'intervention où la famille était présente lors de la réanimation. Cette étude a été reconduite un an après la réanimation à travers la même interview téléphonique suivant le même processus que celle conduite en 2013 chez les mêmes familles. Un an après, les résultats montrent que le syndrome de stress post-traumatique est significativement plus présent dans le groupe témoin que dans le groupe expérimental [48] (Tableau 2).

La prise en charge des familles dont le proche est pris en charge en réanimation paraît donc une évidence dans la mesure où elles sont elles-mêmes impactées par le syndrome de stress post-traumatique.

\section{Soins paramédicaux : facilitateurs et barrières}

Malgré l'incidence élevée du delirium en réanimation, les complications à court terme et le retentissement de la pathologie sur la qualité de vie, le delirium reste sous-évalué comme le montre l'étude d'El Hussein et al. en 2015 [48]. De nombreux autres travaux $[49,50]$ ont également montré le rôle majeur que jouent les paramédicaux dans la prévention et la détection de cette pathologie mais mettent également en lumière certains facteurs contributifs à la sous-détection de cette pathologie. Les barrières et les facilitateurs décrits dans cette partie à travers des résultats d'études soulignent les stratégies nécessaires et les conditions requises à la mise en œuvre d'un travail de dépistage et de prévention de qualité auprès des patients.

\section{Facilitateurs}

- L'amélioration de la confiance en soi des infirmières [51] grâce à une formation continue ou un programme d'entraînement peut permettre d'améliorer le dépistage précoce et les soins auprès des patients en delirium ;

- une stratégie pluridisciplinaire avec des feed-back auprès des pairs et des experts est un facteur de renforcement permettant la consolidation de l'apprentissage [52] ;

- Meijers et al. [53] identifient six facteurs contextuels ayant une relation statistiquement significative avec l'utilisation des données probantes, à savoir le rôle de l'infirmière, l'accès polyvalent aux ressources, le climat organisationnel, le soutien multidimensionnel, le temps consacré 
Tableau 2 Évaluation psychologique à un an des membres de la famille participant à l'étude [47]

\begin{tabular}{|c|c|c|c|c|c|c|}
\hline \multirow[t]{2}{*}{ Résultats } & \multicolumn{3}{|c|}{ Groupe randomisé $(N=408)$} & \multicolumn{3}{|c|}{$\begin{array}{l}\text { Option donnée au membre de la famille } \\
\text { pour assister à la } \operatorname{RCP}(N=408)\end{array}$} \\
\hline & $\begin{array}{l}\text { Groupe } \\
\text { intervention } \\
(N=198)\end{array}$ & $\begin{array}{l}\text { Groupe } \\
\text { contrôle } \\
(N=\mathbf{2 1 0})\end{array}$ & $\begin{array}{l}P \\
\text { value* }^{*}\end{array}$ & Oui $(N=239)$ & Non $(N=169)$ & $\begin{array}{l}P \\
\text { value* }\end{array}$ \\
\hline Score IES, médiane [EI] & $19[7-28]$ & $20[11-35]$ & 0,03 & $18[7-28]$ & $21[11-35]$ & 0,006 \\
\hline Présence de symptômes liés au SSPT, n (\%) & $39(20)$ & $67(32)$ & 0,01 & $52(22)$ & $54(32)$ & 0,06 \\
\hline Score HADS, médiane $[\mathrm{EI}]^{\mathrm{a}}$ & $8[4-13]$ & $10[5-16]$ & 0,41 & $8[4-13]$ & $10[5-16]$ & 0,22 \\
\hline Symptômes d'anxieté, n (\%) & $26(13)$ & $37(18)$ & 0,74 & $33(14)$ & $30(18)$ & 0,85 \\
\hline Symptômes de dépression, n (\%) & $19(10)$ & $32(16)$ & 0,003 & $26(11)$ & $25(15)$ & 0,01 \\
\hline $\begin{array}{l}\text { A vu un psychologue après la réanimation } \\
\text { du patient, } n(\%)^{\mathrm{a}}\end{array}$ & $23(12)$ & $27(13)$ & 0,76 & $27(12)$ & $23(14)$ & 0,59 \\
\hline $\begin{array}{l}\text { A reçu de nouveaux médicaments } \\
\text { psychotiques prescrits après la réanimation } \\
\text { du patient, } \mathrm{n}(\%)^{\mathrm{a}}\end{array}$ & $54(28)$ & $69(34)$ & 0,01 & $66(28)$ & $57(34)$ & 0,006 \\
\hline $\begin{array}{l}\text { Tentative de suicide après la réanimation } \\
\text { du patient, } \mathrm{n}(\%)^{\mathrm{a}}\end{array}$ & $2(1)$ & $5(2)$ & - & $4(2)$ & $3(2)$ & - \\
\hline $\begin{array}{l}\text { Épisode majeur dépressif défini par le score } \\
\text { MINI, n }(\%)^{b}\end{array}$ & $45(23)$ & $64(31)$ & 0,02 & $57(24)$ & $52(31)$ & 0,03 \\
\hline Score ICG, médiane[EI] $]^{\mathrm{a}}$ & $16[9-23]$ & $19[9-28]$ & 0,06 & $15,5[8-23,5]$ & $20[11,5-28,5]$ & 0,01 \\
\hline Présence d'un deuil compliqué, n (\%) & $41(21)$ & $71(36)$ & 0,005 & $53(23)$ & $59(37)$ & 0,003 \\
\hline \multicolumn{7}{|c|}{$\begin{array}{l}\text { La présence d'un SSPT est définie par un score IES }>30 \text {. Les symptomes d'anxieté ou de dépression sont définis par un score HADS } \\
>10 \text {. La présence d'un deuil compliqué est definie par un score ICG }>25 \text {. } \\
\text { RCP Réanimation Cardiopulmonaire, IES échelle d'impact d'évènement (impact of event scale), SSPT syndrome de stress post- } \\
\text { traumatique, EI écart interquartile, HADS questionnaire d'anxiété et de dépression hospitalière (hospital anxiety and depression } \\
\text { scale), MINI mini entretien neuropsychiatrique (mini-international neuropsychiatric interview), ICG inventaire de deuil compliqué } \\
\text { (inventory of complicated grief) }\end{array}$} \\
\hline
\end{tabular}

aux activités de recherche et l'éducation. En mettant en relation le niveau de formation des infirmières et leurs accès aux données de la recherche, cette revue systématique met en correspondance le niveau de formation et l'organisation efficiente du système de la santé ;

- l'utilisation d'outils validés, comme le montre une étude pré/post [49], est nécessaire pour objectiver le delirium qui a tendance à être surestimé par les infirmières de soins intensifs n'utilisant pas d'échelle validée. Cette étude révèle également l'importance de l'utilisation d'un outil diagnostique sur toutes les plages horaires de travail.

\section{Barrières}

- Les connaissances insuffisantes sur le delirium jouent un rôle dans la sous-évaluation du syndrome et par consé- quent son défaut de prise en charge. Ce manque de connaissance concerne : les causes du delirium [54], la comorbidité associée [55], l'importance du dépistage du delirium par des outils validés dans les services [56] ;

- le caractère aléatoire de l'expression du delirium [57] influence l'identification des cas de delirium et engendre une sous-estimation du dépistage des formes hypoactives en l'absence d'outil diagnostique ;

- le delirium est vécu comme un «fardeau » subjectif pour les paramédicaux qui prennent en charge les patients présentant un delirium. McDonnell et Timmins décrivent en 2012 [58] que ce sont les formes hyperactives du delirium, pour lesquelles les patients sont généralement en état d'agitation, difficiles à gérer et peu coopératifs, qui sont les plus retrouvées et considérées comme fardeau par les soignants ; 
- les facteurs institutionnels d'organisation de l'institution peuvent être également un frein. On retrouve le manque de temps de formation, la charge de travail ou encore le manque de personnel comme le démontrent Soja et al. [59].

Les barrières retrouvées dans le dépistage du delirium sont de différents ordres et touchent principalement les connaissances et les attitudes des soignants face au delirium. Le contexte organisationnel peut aussi influencer l'utilisation des connaissances dans la pratique. Le leadership et l'organisation du système de santé sont mis en correspondance avec l'application des recommandations au travers de ces études.

\section{Conclusion}

La sensibilisation des soignants à la pathologie et la mise en lumière des facteurs étiologiques pourraient permettre de dépister le delirium plus précocement et de le gérer indépendamment des autres pathologies grâce à un nouveau référentiel de pratiques [35]. Le dépistage systématique associé à des actions de prévention non pharmacologiques permettra une prise en charge précoce des épisodes de delirium [60].

En prenant en considération l'étiologie de ces risques de morbidité et en agissant sur les facteurs de risque modulables (durée de la sédation, niveau sonore, cycle nycthéméral, etc.), nous apportons un double bénéfice au patient : réduire l'incidence, voire la durée du delirium et prévenir les complications du delirium à court, moyen et long termes, grâce à la prévention par des actions non pharmacologiques associées.

Ces actions s'articulent autour de différents axes :

- la place primordiale faite à l'amélioration des conditions environnementales pour agir sur les facteurs de risque modulables (bruit, lumière, etc.) ;

- le rôle infirmier à investir dans la prévention et le dépistage du delirium (évaluation systématique et stratégies de soins de supports personnalisées) ;

- le besoin de formation continue des paramédicaux identifié comme facilitateur, afin d'améliorer leurs arbres décisionnels de prise en charge ;

- la mise en place de stratégies pluridisciplinaires dans la prise en charge du delirium, décrit comme facilitateur, afin de cibler de façon efficiente les différentes étiologies.

Liens d'intérêts : les auteurs déclarent ne pas avoir de lien d'intérêt.

\section{Références}

1. Association AP, (2004) DSM-IV-TR. Elsevier Masson, $1146 \mathrm{p}$

2. Pipanmekaporn T, Chittawatanarat $\mathrm{K}$, Chaiwat $\mathrm{O}$, Thawitsri $\mathrm{T}$, Wacharasint P, Kongsayreepong S, THAI-SICU Study Group,
(2015) Incidence and risk factors of delirium in multi-center Thai surgical intensive care units: a prospective cohort study. J Intensive Care 3: 53

3. Voyer P, Champoux N, Desrosiers J, Landreville P, McCusker J, Monette J, Savoie M, Richard S, Carmichael PH, (2015) Recognizing acute delirium as part of your routine [RADAR]: a validation study. BMC Nurs 14: 19

4. Poikajärvi S, Salanterä S, Katajisto J, Junttila K, (2017) Validation of finnish neecham confusion scale and nursing delirium screening scale using confusion assessment method algorithm as a comparison scale. BMC Nurs 16: 7

5. Radtke FM, Franck M, Schust S, Boehme L, Pascher A, Bail HJ, Seeling M, Luetz A, Wernecke KD, Heinz A, Spies CD, (2010) A comparison of three scores to screen for delirium on the surgical ward. World J Surg 34: 487-494

6. Ely EW, Margolin R, Francis J, May L, Truman B, Dittus R, Speroff T, Gautam S, Bernard GR, Inouye SK, (2001) Evaluation of delirium in critically ill patients: validation of the Confusion Assessment Method for the Intensive Care Unit (CAM-ICU). Crit Care Med 29: 1370-1379

7. Bergeron N, Dubois MJ, Dumont M, Dial S, Skrobik Y, (2001) Intensive Care Delirium Screening Checklist: evaluation of a new screening tool. Intensive Care Med 27: 859-864

8. Boettger S, Nuñez DG, Meyer R, Richter A, Fernandez SF, Rudiger A, Schubert M, Jenewein J, (2017) Delirium in the intensive care setting: a reevaluation of the validity of the CAM-ICU and ICDSC versus the DSM-IV-TR in determining a diagnosis of delirium as part of the daily clinical routine. Palliat Support Care 8: 675-683

9. Luetz A, Heymann A, Radtke FM, Chenitir C, Neuhaus U, Nachtigall I, von Dossow V, Marz S, Eggers V, Heinz A, Wernecke KD, Spies CD, (2010) Different assessment tools for intensive care unit delirium: which score to use? Crit Care Med 38: 409-418

10. Girard TD, Pandharipande PP, Ely EW, (2008) Delirium in the intensive care unit. Crit Care 12: S3

11. Newman MW, O’Dwyer LC, Rosenthal L, (2015) Predicting delirium: a review of risk-stratification models. Gen Hosp Psychiatry 37: $408-413$

12. Arend E, Christensen M, (2009) Delirium in the intensive care unit: a review. Nurs Crit Care 14: 145-154

13. Zaal IJ, Spruyt CF, Peelen LM, van Eijk MMJ, Wientjes R, Schneider MME, Kesecioglu J, Slooter AJC, (2013) Intensive care unit environment may affect the course of delirium. Intensive Care Med 39: 481-488

14. Girard TD, Pandharipande PP, Ely EW, (2008) Delirium in the intensive care unit. Crit Care Lond Engl 12: S3

15. Mehta S, Cook D, Devlin JW, Skrobik Y, Meade M, Fergusson D, Herridge M, Steinberg M, Granton J, Ferguson N, Tanios M, Dodek P, Fowler R, Burns K, Jacka M, Olafson K, Mallick R, Reynolds S, Keenan S, Burry L, for the SLEAP Investigators and the Canadian Critical Care Trials Group, (2014) Prevalence, risk factors, and outcomes of delirium in mechanically ventilated adults. Crit Care Med 43: 557-566

16. Oldham M, Pisani MA, (2015) Sedation in critically ill patients. Crit Care Clin 31: 563-587

17. Jackson P, Khan A, (2015) Delirium in critically ill patients. Crit Care Clin 31: 589-603

18. van den Boogaard M, Pickkers P, Slooter AJC, Kuiper MA, Spronk PE, van der Voort PHJ, van der Hoeven JG, Donders R, van Achterberg T, Schoonhoven L, (2012) Development and validation of PRE-DELIRIC (PREdiction of DELIRium in ICu patients) delirium prediction model for intensive care patients: observational multicentre study. The BMJ 344: e420

19. Chanques G, Payen JF, Mercier G, de Lattre S, Viel E, Jung B, Cissé M, Lefrant JY, Jaber S, (2009) Assessing pain in nonintubated critically ill patients unable to self report: an adaptation of the Behavioral Pain Scale. Intensive Care Med 35: 2060-2067 
20. Kamdar BB, Niessen T, Colantuoni E, King LM, Neufeld KJ, Bienvenu OJ, Rowden AM, Collop NA, Needham DM, (2015) Delirium transitions in the medical ICU: exploring the role of sleep quality and other factors. Crit Care Med 43: 135

21. Weinhouse GL, (2015) When perception may not meet reality: sleep and delirium in the ICU. Crit Care Med 43: 248-249

22. Puntillo KA, Arai S, Cohen NH, Gropper MA, Neuhaus J, Paul SM, Miaskowski C, (2010) Symptoms experienced by intensive care unit patients at high risk of dying. Crit Care Med 38: $2155-2160$

23. Ouimet S, Kavanagh BP, Gottfried SB, Skrobik Y, (2007) Incidence, risk factors and consequences of ICU delirium. Intensive Care Med 33: 66-73

24. Pauley E, Lishmanov A, Schumann S, Gala GJ, van Diepen S, Katz JN, (2015) Delirium is a robust predictor of morbidity and mortality among critically ill patients treated in the cardiac intensive care unit. Am Heart J 170: 79-86, 86.e1

25. Lee E, Kim J, (2014) Cost-benefit analysis of a delirium prevention strategy in the intensive care unit. Nurs Crit Care 21: 367-373

26. Rothenhäusler HB, Ehrentraut S, Stoll C, Schelling G, Kapfhammer HP, (2001) The relationship between cognitive performance and employment and health status in long-term survivors of the acute respiratory distress syndrome: results of an exploratory study. Gen Hosp Psychiatry 23: 90-96

27. Sakuramoto H, Subrina J, Unoki T, Mizutani T, Komatsu H, (2015) Severity of delirium in the ICU is associated with short term cognitive impairment. A prospective cohort study. Intensive Crit Care Nurs 31: 250-257

28. McCusker J, Cole MG, Voyer P, Monette J, Champoux N, Ciampi A, Vu M, Belzile E, (2014) Six-month outcomes of co-occurring delirium, depression, and dementia in long-term care. J Am Geriatr Soc 62: 2296-2302

29. Jackson JC, Pandharipande PP, Girard TD, Brummel NE, Thompson JL, Hughes CG, Pun BT, Vasilevskis EE, Morandi A, Shintani AK, Hopkins RO, Bernard GR, Dittus RS, Ely EW, (2014) Depression, posttraumatic stress disorder, and functional disability in survivors of critical illness: results from the BRAIN ICU (Bringing to light the Risk Factors And Incidence of Neuropsychological dysfunction in ICU survivors) Investigation: a longitudinal cohort study. Lancet Respir Med 2: 369-379

30. Langan C, Sarode DP, Russ TC, Shenkin SD, Carson A, Maclullich AMJ, (2017) Psychiatric symptomatology after delirium: a systematic review. Psychogeriatrics 17: 327-335

31. Su X, Meng ZT, Wu XH, Cui F, Li HL, Wang DX, Zhu X, Zhu SN, Maze M, Ma D, (2016) Dexmedetomidine for prevention of delirium in elderly patients after non-cardiac surgery: a randomised, double-blind, placebo-controlled trial. The Lancet 388: 1893-1902

32. Reade MC, Eastwood GM, Bellomo R, Bailey M, Bersten A, Cheung B, Davies A, Delaney A, Ghosh A, Haren F van, Harley N, Knight D, McGuiness S, Mulder J, O'Donoghue S, Simpson N, Young P, (2016) Effect of dexmedetomidine added to standard care on ventilator-free time in patients with agitated delirium: a randomized clinical trial. JAMA 315: 1460-1468

33. Barr J, Fraser GL, Puntillo K, Ely EW, Gélinas C, Dasta JF, Davidson JE, Devlin JW, Kress JP, Joffe AM, Coursin DB, Herr DL, Tung A, Robinson BRH, Fontaine DK, Ramsay MA, Riker RR, Sessler CN, Pun B, Skrobik Y, Jaeschke R, American College of Critical Care Medicine, (2013) Clinical practice guidelines for the management of pain, agitation, and delirium in adult patients in the intensive care unit. Crit Care Med 41: 263-306

34. Davidson JE, Winkelman C, Gélinas C, Dermenchyan A, (2015) Pain, agitation, and delirium guidelines: nurses' involvement in development and implementation. Crit Care Nurse 35: 17-31; quiz 32

35. Barnes-Daly MA, Phillips G, Ely EW, (2017) Improving hospital survival and reducing brain dysfunction at seven California com- munity hospitals: implementing PAD guidelines via the ABCDEF bundle in 6,064 patients. Crit Care Med 45: 171-178

36. Trogrlić Z, van der Jagt M, Bakker J, Balas MC, Ely EW, van der Voort PH, Ista E, (2015) A systematic review of implementation strategies for assessment, prevention, and management of ICU delirium and their effect on clinical outcomes. Crit Care 15: 157

37. Marra A, Ely EW, Pandharipande PP, Patel MB, (2017) The ABCDEF bundle in critical care. Crit Care Clin 33: 225-243

38. Kalfon P, Mimoz O, Auquier P, Loundou A, Gauzit R, Lepape A, Laurens J, Garrigues B, Pottecher T, Mallédant Y, (2010) Development and validation of a questionnaire for quantitative assessment of perceived discomforts in critically ill patients. Intensive Care Med 36: 1751-1758

39. Puntillo K, Nelson JE, Weissman D, Curtis R, Weiss S, Frontera J, Gabriel M, Hays R, Lustbader D, Mosenthal A, Mulkerin C, Ray D, Bassett R, Boss R, Brasel K, Campbell M, (2014) Palliative care in the ICU: relief of pain, dyspnea, and thirst - A report from the IPAL-ICU Advisory Board. Intensive Care Med 40: $235-248$

40. Lang EV, Hatsiopoulou O, Koch T, Berbaum K, Lutgendorf S, Kettenmann E, Logan H, Kaptchuk TJ, (2005) Can words hurt? Patient-provider interactions during invasive procedures. Pain 114: 303-309

41. Rainville P, Hofbauer RK, Paus T, Duncan GH, Bushnell MC, Price DD, (1999) Cerebral mechanisms of hypnotic induction and suggestion. J Cogn Neurosci 11: 110-125

42. Rainville P, Hofbauer RK, Bushnell MC, Duncan GH, Price DD, (2002) Hypnosis modulates activity in brain structures involved in the regulation of consciousness. J Cogn Neurosci 14: 887-901

43. Chooi CSL, Nerlekar R, Raju A, Cyna AM, (2011) The effects of positive or negative words when assessing postoperative pain. Anaesth Intensive Care 39: 101-106

44. Black P, Boore JRP, Parahoo K, (2011) The effect of nursefacilitated family participation in the psychological care of the critically ill patient. J Adv Nurs 67: 1091-1101

45. Huggins EL, Bloom SL, Stollings JL, Camp M, Sevin CM, Jackson JC, (2016) A clinic model: post-intensive care syndrome and post-intensive care syndrome-family. AACN Adv Crit Care 27: 204-211

46. Partridge JSL, Martin FC, Harari D, Dhesi JK, (2013) The delirium experience: what is the effect on patients, relatives and staff and what can be done to modify this? Int J Geriatr Psychiatry 28: 804-812

47. Jabre P, Belpomme V, Azoulay E, Jacob L, Bertrand L, Lapostolle F, Tazarourte K, Bouilleau G, Pinaud V, Broche C, Normand D, Baubet T, Ricard-Hibon A, Istria J, Beltramini A, Alheritiere A, Assez N, Nace L, Vivien B, Turi L, Launay S, Desmaizieres M, Borron SW, Vicaut E, Adnet F, (2013) Family presence during cardiopulmonary resuscitation. N Engl J Med 368: 1008-1018

48. El Hussein M, Hirst S, Salyers V, (2015) Factors that contribute to underrecognition of delirium by registered nurses in acute care settings: a scoping review of the literature to explain this phenomenon. J Clin Nurs 24: 906-915

49. Reade MC, Eastwood GM, Peck L, Bellomo R, Baldwin I, (2011) Routine use of the Confusion Assessment Method for the Intensive Care Unit (CAM-ICU) by bedside nurses may underdiagnose delirium. Crit Care Resusc 13: 217-224

50. Pun BT, Devlin JW, (2013) Delirium monitoring in the ICU: strategies for initiating and sustaining screening efforts. Semin Respir Crit Care Med 34: 179-188

51. Akechi T, Ishiguro C, Okuyama T, Endo C, Sagawa R, Uchida M, Furukawa TA, (2010) Delirium training program for nurses. Psychosomatics 51: 106-111

52. Yanamadala M, Wieland D, Heflin MT, (2013) Educational interventions to improve recognition of delirium: a systematic review. J Am Geriatr Soc 61: 1983-1993 
53. Meijers JMM, Janssen MAP, Cummings GG, Wallin L, Estabrooks CA, YG Halfens R, (2006) Assessing the relationships between contextual factors and research utilization in nursing: systematic literature review. J Adv Nurs 55: 622-635

54. Gesin G, Russell BB, Lin AP, Norton HJ, Evans SL, Devlin JW, (2012) Impact of a delirium screening tool and multifaceted education on nurses' knowledge of delirium and ability to evaluate it correctly. Am J Crit Care 21: e1-e11

55. Flagg B, Cox L, McDowell S, Mwose JM, Buelow JM, (2010) Nursing identification of delirium. Clin Nurse Spec CNS 24: 260-266

56. Forsgren LM, Eriksson M, (2010) Delirium--awareness, observation and interventions in intensive care units: a national survey of Swedish ICU head nurses. Intensive Crit Care Nurs 26: 296-303
57. Vollmer CM, Bond J, Eden BM, Resch DS, Fulk L, Robinson S, Hughes LE, (2010) Incidence, prevalence, and under-recognition of delirium in urology patients. Urol Nurs 30: 235-241, 254

58. McDonnell S, Timmins F, (2012) A quantitative exploration of the subjective burden experienced by nurses when caring for patients with delirium. J Clin Nurs 21: 2488-2498

59. Soja SL, Pandharipande PP, Fleming SB, Cotton BA, Miller LR, Weaver SG, Lee BT, Ely EW, (2008) Implementation, reliability testing, and compliance monitoring of the Confusion Assessment Method for the Intensive Care Unit in trauma patients. Intensive Care Med 34: 1263-1268

60. Bailey PP, Miller RR, Clemmer TP, (2009) Culture of early mobility in mechanically ventilated patients. Crit Care Med 37: S429-S435 